\title{
Fine mapping of eight psoriasis susceptibility loci
}

\author{
Sayantan Das ${ }^{1}$, Philip E Stuart ${ }^{2}$, Jun Ding ${ }^{1,3}$, Trilokraj Tejasvi ${ }^{2}$, Yanming $\mathrm{Li}^{1}$, Lam C Tsoi ${ }^{1}$, Vinod Chandran ${ }^{4}$, \\ Judith Fischer ${ }^{5}$, Cynthia Helms ${ }^{6}$, Kristina Callis Duffin ${ }^{7}$, John J Voorhees ${ }^{2}$, Anne M Bowcock ${ }^{6}$, \\ Gerald G Krueger ${ }^{7}$, G Mark Lathrop ${ }^{5}$, Rajan P Nair ${ }^{2}$, Proton Rahman ${ }^{8}$, Goncalo R Abecasis ${ }^{\star, 1}$, \\ Dafna Gladman ${ }^{4,9}$ and James T Elder ${ }^{\star, 2,10}$
}

Previous studies have identified 41 independent genome-wide significant psoriasis susceptibility loci. After our first psoriasis genome-wide association study, we designed a custom genotyping array to fine-map eight genome-wide significant susceptibility loci known at that time (IL23R, IL13, IL12B, TNIP1, MHC, TNFAIP3, IL23A and RNF114) enabling genotyping of 2269 single-nucleotide polymorphisms (SNPs) in the eight loci for 2699 psoriasis cases and 2107 unaffected controls of European ancestry. We imputed these data using the latest 1000 Genome reference haplotypes, which included both indels and SNPs, to increase the marker density of the eight loci to 49239 genetic variants. Using stepwise conditional association analysis, we identified nine independent signals distributed across six of the eight loci. In the major histocompatibility complex (MHC) region, we detected three independent signals at rs114255771 $\left(P=2.94 \times 10^{-74}\right)$, rs6924962 $\left(P=3.21 \times 10^{-19}\right)$ and rs892666 $\left(P=1.11 \times 10^{-10}\right)$. Near IL12B we detected two independent signals at rs62377586 $\left(P=7.42 \times 10^{-16}\right)$ and rs918518 $\left(P=3.22 \times 10^{-11}\right)$. Only one signal was observed in each of the TNIP1 (rs17728338; $\left.P=4.15 \times 10^{-13}\right), I L 13$ (rs1295685; $P=1.65 \times 10^{-7}$ ), IL23A (rs61937678; $P=1.82 \times 10^{-7}$ ) and TNFAIP3 (rs642627; $P=5.90 \times 10^{-7}$ ) regions. We also imputed variants for eight HLA genes and found that SNP rs114255771 yielded a more significant association than any HLA allele or amino-acid residue. Further analysis revealed that the HLA-C*06-B*57 haplotype tagged by this SNP had a significantly higher odds ratio than other $H L A-C * 06$-bearing haplotypes. The results demonstrate allelic heterogeneity at IL12B and identify a high-risk MHC class I haplotype, consistent with the existence of multiple psoriasis effectors in the MHC. European Journal of Human Genetics (2015) 23, 844-853; doi:10.1038/ejhg.2014.172; published online 3 September 2014

\section{INTRODUCTION}

Psoriasis (OMIM no. 177900) is a common immune-mediated disease characterized by painful, red scaly patches of skin. Psoriasis affects $0.2-2 \%$ of individuals and disease etiology likely involves multiple genetic and environmental factors. Psoriatic patients can develop an inflammatory arthritis and are at an elevated risk of comorbidities such as heart disease, metabolic syndrome and cardiovascular disease; ${ }^{1}$ they also exhibit increased risk for other immune-mediated disorders such as Crohn's disease, celiac disease, ankylosing spondylitis, leprosy, multiple sclerosis, systemic sclerosis and rheumatoid arthritis. ${ }^{2}$ Genome-wide association studies $(\text { GWAS })^{3-7}$ have identified more than 41 independent signals in 36 disease-susceptibility regions ${ }^{3,7,8}$ for individuals of European ancestry, although much of the genetic contribution to psoriasis remains unexplained.

The strongest psoriasis susceptibility locus lies within the major histocompatibility complex (MHC) and is termed PSORS1 (psoriasis susceptibility locus 1 ). Other important contributors include IL12B, IL23R, IL23A, TNFAIP3, IL13, RNF114 and TNIP1. ${ }^{4-6,9}$ Although GWAS provides an efficient strategy for identifying disease susceptibility loci, detailed assessments of the contribution of these loci to disease require systematic investigation of common and rare variants in each locus. Previous fine-mapping studies in psoriasis have focused on the MHC, helping localize the primary risk locus ${ }^{10,11}$ and identify multiple psoriasis susceptibility alleles. ${ }^{8,12}$

Using a combination of genotyping and imputation, ${ }^{13}$ we examined in detail the genetic variation in each of the eight psoriasis susceptibility regions (MHC, IL12B, IL23A, IL23R, TNIP1, TNFAIP3, IL13 and RNF114) in a large sample of subjects of European ancestry, over $97 \%$ of whom were not previously subjected to fine-mapping. We sought to identify the variant with strongest association in each locus as well as to find out whether multiple variants might be independently associated in the same locus.

\section{MATERIALS AND METHODS}

Samples and markers

We collected 5067 samples of European ancestry from five collaborating centers (Table 1). Boundaries for five of the fine-mapping regions were defined to include all genes of interest in their entirety and all single-nucleotide

${ }^{1}$ Department of Biostatistics, Center for Statistical Genetics, University of Michigan, Ann Arbor, MI, USA; ${ }^{2}$ Department of Dermatology, University of Michigan, Ann Arbor, MI, USA; ${ }^{3}$ Laboratory of Genetics, National Institute on Aging, National Institutes of Health, Baltimore, MD, USA; ${ }^{4}$ Division of Rheumatology, Department of Medicine, University of Toronto, Toronto, ON, Canada; ${ }^{5}$ Centre National de Génotypage, Institut Génomique, Commissariat à l'Énergie Atomique, Evry, France; ${ }^{6}$ National Heart and Lung Institute, Imperial College London, London, UK; ${ }^{7}$ Department of Dermatology, University of Utah, Salt Lake City, UT, USA; ${ }^{8}$ Department of Medicine, Memorial University, St John's, NL, Canada; ${ }^{9}$ Psoriatic Arthritis Program, Centre for Prognosis Studies in the Rheumatic Diseases, Toronto Western Research Institute, Toronto, ON, Canada; ${ }^{10}$ Ann-Arbor Veteran Affairs Hospital, Ann Arbor, MI, USA

${ }^{*}$ Correspondence: Dr GR Abecasis, Department of Biostatistics, Center for Statistical Genetics, University of Michigan, M4614 SPH I Tower, 1415 Washington Heights, Ann Arbor, MI 48109-2029, USA. Tel: +1 734763 4901; Fax: +1 734615 8322; E-mail: goncalo@umich.edu

or Dr JT Elder, Department of Biostatistics, Center for Statistical Genetics, University of Michigan Medical School, University of Michigan, $7412 \mathrm{~A}$ Medical Science Building I, 1301 East Catherine Street, Ann Arbor, MI 48109-5675, USA. Tel: +1 734647 8070; Fax: +1 734615 7277; E-mail: jelder@umich.edu

Received 3 October 2013; revised 3 June 2014; accepted 6 June 2014; published online 3 September 2014 
Table 1 Summary of samples based on cohort

\begin{tabular}{lcccc}
\hline Cohort & $\begin{array}{c}\text { Total } \\
\text { samples }\end{array}$ & $\begin{array}{c}\text { Cases } \\
(\%)\end{array}$ & $\begin{array}{c}\text { Controls } \\
(\%)\end{array}$ & $\begin{array}{c}\text { No. of samples in } \\
\text { Tsoi et al }^{7}(\%)\end{array}$ \\
\hline St Louis & 258 & $4(1.5)$ & $254(98.5)$ & $0(0)$ \\
Michigan & 2350 & $986(42)$ & $1364(58)$ & $85(3.6)$ \\
Utah & 490 & $250(51)$ & $240(49)$ & $0(0)$ \\
Toronto & 1186 & $502(42)$ & $684(58)$ & $2(0.1)$ \\
Newfoundland & 783 & $470(60)$ & $313(40)$ & $58(7.4)$ \\
Total & 5067 & $2212(44)$ & $2855(56)$ & $145(2.9)$ \\
\hline
\end{tabular}

polymorphisms (SNPs) with association $P$-values in the CASP-GWAS ${ }^{4}$ passing a predefined threshold (see Supplementary Table 2 for details). For TNFAIP3, we considered a broader region (476 kb on chr6.hg19.g.138001712-138478136) that covers the candidate disease risk regions for several autoimmune diseases, including psoriasis, psoriatic arthritis (PsA), rheumatoid arthritis and systemic lupus erythematosus. In total, 408 SNPs were picked from this region. The MHC region was defined to include four possible MHC disease loci - a 224-kb candidate interval for PSORS1, ${ }^{10}$ a secondary locus not in linkage disequilibrium (LD) with PSORS1, which was tagged by rs2022544 chr6.hg19.g.32321004G $>$ A near $H L A-D R A,{ }^{4}$ and two possible PsA loci tagged by rs12175489 chr6.hg19.g.31377587G >A in MICA and rs3130180 chr6.hg19.g.33020286G >C in HLA-DPA1/HLA-DPB1 (unpublished data). As LD is so extensive in the MHC region and candidate intervals have not been determined for the secondary loci, our fine-mapping region was designed to include all but $250 \mathrm{~kb}$ of the classic MHC. We designed an Illumina (San Diego, CA, USA) iSelect custom genotyping array with 2269 tagging SNPs in the eight regions and 5463 SNPs outside the eight regions. All SNPs from two MHC panels designed for the Illumina Golden Gate platform (mapping panel GS0006599-OPA and exon-centric panel GS0006598-OPA) falling within the $3.37 \mathrm{Mb}$ candidate region (chr6.hg19.g.29882021-33252022) were included. SNPs were removed if they had Illumina Infinium design scores $<0.5$ or if they had an Infinium I design (ie, A/T or C/G SNPs requiring two bead assays for one genotyping). In addition, 31 SNPs in this region were included to tag HLA-Cw6-bearing risk haplotypes. ${ }^{10}$ For the other seven regions, all SNPs from the August 2009 release of the 1000 Genomes project with $\mathrm{MAF} \geq 0.01$ in $\mathrm{CEU}$ samples were considered as candidates. After excluding SNPs with Infinium design scores $<0.6$ or with Infinium I designs, tagging $\mathrm{SNPs}^{14}$ were selected using an $\mathrm{LD}$ value of $r^{2} \geq 0.8$ as a threshold. A backup SNP was added for every SNP tagging more than five other variants to guard against genotyping failure, and all SNPs in moderate to high LD $\left(r^{2} \geq 0.5\right)$ with the most strongly associated SNP in each region were unconditionally included. Eighty-six additional SNPs for these seven regions were also genotyped, including 63 high-priority variants (5'-UTR, $3^{\prime}$-UTR, essential splice site, nonsynonymous and synonymous coding, gained stop codon) from the 1000 Genomes call set and 23 SNPs within the TNFAIP3 region that are associated with other autoimmune disorders. ${ }^{15,16}$

Samples with $<95 \%$ genotyping rate or with extreme heterozygosity were excluded from analysis. Sixty-three samples that were duplicates or close relatives of other samples in the study (identified with the program relativeFinder ${ }^{17}$ ) and 185 samples with evidence of non-European ancestry (identified with principal component analysis; Supplementary Figure 1) were also excluded from further analysis. A total of 4806 samples (2699 cases and 2107 controls) were analyzed. Genotypes and phenotypes for the 4806 samples and 7732 markers analyzed in this study have been submitted to the NCBI dbGaP database at http://www.ncbi.nlm.nih.gov/gap (dbGaP accession number: phs000019.v1.p1).

\section{Genotype imputation}

We used information on patterns of haplotype variation available in European ancestry (EUR) samples in the 1000 Genomes Project Phase I release version $3^{13}$ to increase the density of the variants in the eight regions. We first used $\mathrm{MaCH}^{18}$ to perform the phasing of the genotype data and then used Minimac $^{19}$ for imputation. We restricted analysis of genetic variants in the eight known regions to those that were genotyped on the array or could be imputed with acceptable quality (estimated $r^{2}$ between the imputed dosage and true genotypes $\geq 0.3$ ).

We used SNP2HLA ${ }^{20}$ and a data set collected by the Type 1 Diabetes Genetics Consortium (T1DGC) as a reference panel to impute 8961 variants in the MHC region (126 classical HLA 2-digit alleles, 298 HLA 4-digit alleles, 1276 polymorphic amino-acid residues of these classical alleles and 7261 SNPs). Alleles and amino acids were imputed for eight HLA genes that were genotyped in the T1DGC reference panel (HLA-A, -B, -C, -DPA1, -DPB1, $-D Q A 1,-D Q B 1$ and $-D R B 1)$. These variants were merged with 40074 SNPs and indel variants in the MHC region that were imputed using the 1000 Genomes data set as a reference panel, but were not part of the T1DGC reference set, giving a combined data set of $49035 \mathrm{MHC}$ variants for analysis.

\section{Association analysis}

We used a variance component mixed-model association test implemented in the EMMAX software package to identify disease-associated variants. ${ }^{21}$ Variance component models correct for a wide range of potential problems, including population structure, by modeling pairwise genotype similarity between individuals (4265 independent markers outside the 41 known psoriasis susceptibility loci ${ }^{22}$ were used to estimate kinship coefficients). We began with an unconditional analysis of all genotyped and well-imputed variants in the eight regions, selecting the most statistically significant signal in each region that survived Bonferroni correction for the total number of variants tested. We then used a stepwise forward selection method to select iteratively additional signals, conditional on signals from the previous iterations.

Genomic control inflation factors $\left(\lambda_{\mathrm{GC}}\right)$ were calculated for each round of conditional analysis based on $P$-values at a set of 4265 independent markers outside the 41 known psoriasis susceptibility loci. ${ }^{22}$ We estimated odds ratios (ORs) for the associated markers using a logistic regression model that included, as ancestry covariates, coordinates obtained from PLINK ${ }^{23}$ for the top 10 dimensions of a multidimensional scaling analysis of an identity-bystate distance matrix based on variants outside the eight regions. We estimated the variance in liability (locus-specific heritability) ${ }^{24}$ that can be explained by the significant SNPs as determined under a liability threshold model ${ }^{25}$ (assuming that the multiple loci have an additive effect on the risk of psoriasis). We used the prevalence of the disease as 0.02 and calculated risk ratios from our estimated ORs using an iterative approach. ${ }^{26}$ We used ANNOVAR $^{27}$ for functional annotation of our significant signals and their proxies ( $r^{2} \geq 0.9$ in 1000 Genomes Project EUR samples).

For association analysis of the MHC variant data set that includes HLA alleles and amino acids, we implemented a forward-selection regression method using a logistic model, controlling for the ancestry covariates. Significance was defined by Bonferroni correction for 49035 variants tested. We also compared the log odds of the $H L A-C^{*} 06-B^{*} 57$ haplotype to other $H L A-C^{\star} 06$-bearing haplotypes in a logistic model and used bootstrap methods to determine significance of the difference in log ORs.

\section{RESULTS}

Using 2269 genotyped SNPs among the eight regions, there were 49239 SNPs and insertions and deletions (indels) with estimated imputation quality $r^{2} \geq 0.3$ (Supplementary Table 1 ). Around $70 \%$ of the well-imputed 1000 Genomes variants (estimated imputation quality $r^{2} \geq 0.3$ ) were common variants (minor allele frequency $\geq 0.05$ ) (Supplementary Figure 2). Variants in the MHC region account for $>80 \%$ of the imputed variants. For association analyses, we used a Bonferroni significance threshold of $1.01 \times 10^{-6}(=0.05 /$ 49239) to correct for multiple testing.

We identified five significant signals from an initial unconditional analysis of the eight loci and identified four additional signals after conditional analyses (Table 2). Taken together, they explain $12.09 \%$ of the variance in liability for psoriasis or $\sim 18.3 \%$ of its estimated heritability. Among the nine independent signals, three were from the MHC, two were from IL12B and one each was from IL13, IL23A, 
TNFAIP3 and TNIP1. The $\lambda_{\mathrm{GC}}$ values for the unconditional round and two conditional rounds were 1.03, 1.02 and 1.02, respectively. The Q-Q plots are shown in Supplementary Figure 3.

The most strongly associated variant (rs114255771 chr6.hg19. g.31316699G $>$ A) was situated between HLA-C and HLA-B $\left(P\right.$-value $=2.94 \times 10^{-74} ; \quad \mathrm{OR}=4.04 ;$ variance explained $\left(V_{\mathrm{e}}\right)=$ $5.23 \%$; risk allele frequency in case/control $\left.\left(f_{\text {ratio }}\right)=0.19 / 0.08\right)$. The two additional independent signals in the MHC region were rs6924962 chr6.hg19.g.31358302C $>\mathrm{T}\left(P\right.$-value $=3.21 \times 10^{-19}$; $\left.\mathrm{OR}=1.82 ; \quad V_{\mathrm{e}}=1.64 \% ; f_{\text {ratio }}=0.23 / 0.16\right)$ near the MICA gene, and rs892666 chr6.hg19.g.29924728C $>\mathrm{T} \quad\left(P\right.$-value $=1.11 \times 10^{-10}$; $\left.\mathrm{OR}=1.48 ; V_{\mathrm{e}}=1.10 \% ; f_{\text {ratio }}=0.70 / 0.66\right)$ near $H L A-A$ (Figure 1$)$. The three MHC signals together accounted for $7.97 \%$ of the total variance in disease liability.

IL12B had two independent signals - rs62377586 chr5.hg19. g.158766022G $>$ A $\quad\left(P\right.$-value $=7.42 \times 10^{-16} ; \quad O R=1.43 ; \quad V_{\mathrm{e}}=0.9 \%$; $\left.f_{\text {ratio }}=0.74 / 0.67\right) \quad$ and $\quad$ rs918518 chr5.hg19.g.158826493C $>$ T $\left(P\right.$-value $=3.22 \times 10^{-11} ; \quad$ OR $\left.=1.44 ; \quad V_{\mathrm{e}}=0.74 \% ; \quad f_{\text {ratio }}=0.28 / 0.21\right)$ - accounting for $1.64 \%$ of variance in liability. Figure 2 shows the regional association plots for the $I L 12 B$ region for the unconditional and conditional analyses.

Signals were also detected in the IL13, TNIP1, TNFAIP3 and IL23A loci, respectively, at rs1295685 chr5.hg19.g.131996445C > T $\left(P\right.$-value $=1.65 \times 10^{-7} ; \quad$ OR $\left.=1.31 ; \quad V_{\mathrm{e}}=0.39 \% ; \quad f_{\text {ratio }}=0.83 / 0.79\right)$, rs17728338 chr5.hg19.g.150478318G $>$ A $\quad\left(P\right.$-value $=4.15 \times 10^{-13}$; $\left.\mathrm{OR}=1.81 ; \quad V_{\mathrm{e}}=0.59 \% ; \quad f_{\text {ratio }}=0.09 / 0.05\right), \quad$ rs642627chr6.hg19.g. $138206783 \mathrm{G}>\mathrm{A} \quad\left(P\right.$-value $=5.90 \times 10^{-7} ; \quad \mathrm{OR}=1.26 ; \quad V_{\mathrm{e}}=0.34 \%$; $\left.f_{\text {ratio }}=0.31 / 0.26\right) \quad$ and $\quad$ rs61937678 chr12.hg19.g.56653695T $>C$ $\left(P\right.$-value $=1.82 \times 10^{-7} ; \quad$ OR $\left.=1.58 ; \quad V_{\mathrm{e}}=1.16 \% ; \quad f_{\text {ratio }}=0.80 / 0.77\right)$. Taken together, these signals explain $2.48 \%$ of the variance in liability (Figure 3). The IL23A region did not have a significant hit in the unconditional round (minimum $P$-value $=1.53 \times 10^{-6}$ at rs61937678 with Bonferroni-adjusted $P$-value cutoff $\left.=1.01 \times 10^{-6}\right)$. However, the same SNP reached significance in an analysis adjusting for rs114255771 (MHC), rs62377586 (IL12B), rs1295685 (IL13), rs17728338 (TNIP1) and rs642627 (TNFAIP3).

We compared our results with those from a recent meta-analysis of GWAS and Immunochip data for psoriasis ${ }^{7}$ (Table 3). As assessed using phase I 1000 genomes data set, our primary signal in the MHC (rs114255771) exhibited complete LD $\left(r^{2}=1\right)$ with rs4406273 chr6.hg19.g.31266090G > A. This SNP was the primary hit in that meta-analysis and also happens to be an excellent surrogate for HLA$C w^{*} 0602$, the leading candidate for the PSORS1 risk allele ${ }^{10,11}$ (unpublished data). Our secondary MHC signal (rs6924962) is in close proximity $(\sim 8.9 \mathrm{~kb})$ to and exhibits moderately strong LD $\left(r^{2}=0.56\right)$ with the secondary MHC signal from the GWASImmunochip meta-analysis (rs13437088 chr6.hg19.g.3135511T >C).

We also compared our results for the MHC region with other finemapping studies of the same region ${ }^{8,12}$ (Figure 4). Although the primary signals agree with each other, the conditional signals of similar rank are not in high LD with each other. Our secondary signal (rs6924962) exhibits moderate LD with the fifth signal rs66609536 chr6.hg19.g.31362120G $>$ A $\left(r^{2}=0.56\right)$ in Knight et al ${ }^{8}$ and the third signal rs13437088 chr6.hg19.g.31355119T $>C\left(r^{2}=0.56\right)$ in Feng et al. ${ }^{12}$

We identified the same variant near IL13 (rs1295685) that was identified in the GWAS-Immunochip meta-analysis, and our primary signals in TNIP1 (rs17728338), TNFAIP3 (rs642627) and IL12B (rs62377586) are in strong LD with the top markers for each locus in that analysis (rs2233278 chr5.hg19.g.150467189C $>\mathrm{G}\left(r^{2}=0.96\right)$, rs582757 chr6.hg19.g.138197824A $>\mathrm{G} \quad\left(r^{2}=0.97\right)$ and $\mathrm{rs} 4379175$ 



Figure 1 Regional association plot for MHC. Association plots for MHC region in the unconditional round, the first conditional round and the second conditional round are shown in (a), (b) and (c), respectively. The horizontal axis represents the base position on chromosome 6 (from 27 to $34 \mathrm{Mb}$ on build 37). The left-vertical axis represents the negative log (base 10) of the $P$-value in the corresponding round (denoted by the circles) and the right-vertical axis represents the recombination rate (in $\mathrm{cM} / \mathrm{Mb}$ ) (denoted by the blue lines). The color coding for the circles is used to represent the linkage disequilibrium with the most significant SNP in the region in each round. 



Figure 2 Regional association plot for $I L 12 B$. Association plots for the $I L 12 B$ region in the unconditional round and the first conditional round are shown in (a) and (b), respectively. The horizontal axis represents the base position on chromosome 5 (from 158.7 to $159 \mathrm{Mb}$ on build 37). The left-vertical axis represents the negative log (base 10) of the $P$-value in the corresponding round (denoted by the circles) and the right-vertical axis represents the recombination rate (in $\mathrm{cM} / \mathrm{Mb}$ ) (denoted by the blue lines). The color coding for the circles is used to represent the linkage disequilibrium with the most significant SNP in the region in each round.

chr5.hg19.g.158804928G $>\mathrm{T}\left(r^{2}=0.84\right)$, respectively). The signal at rs61937678 in the IL23A region is in physical proximity ( $\sim 96.51 \mathrm{~kb})$ of the signal detected at rs2066819 chr12.hg19.g.56750204G $>$ A in the meta-analysis, although the LD between these two SNPs is nominal $\left(r^{2}=0.2\right)$.

We mined the imputed data set for potential causal alleles by examining genome-wide significant variants and their near-perfect proxies ( $r^{2}>0.9$ in 1000 Genomes Project EUR samples); the results are summarized in Supplementary Table 3. Using ANNOVAR, ${ }^{27}$ we found that, of the 39 examined variants, only one (rs20541
chr5.hg19.g.13199596T >A) is exonic. SNP rs20541 is a nonsynonymous SNP in IL13 that is in strong LD $\left(r^{2}=0.97\right)$ with our best IL13 association signal (SNP rs1295685), located in the $3^{\prime}$-UTR of IL13. Nearly all of the remaining 38 variants are either intergenic or intronic based on known genes in the UCSC ${ }^{28}$ and $\operatorname{RefSeq}^{29}$ databases. To assess the possible functional roles of non-protein-coding variants in our data, we used $\mathrm{ENCODE}^{30}$ RNA-seq data to assess transcribed regions as well as ENCODE $^{30}$ data and chromHMM ${ }^{31}$ predictions to categorize the noncoding variants into enhancers, repressors, promoters, insulators, and so on 
a

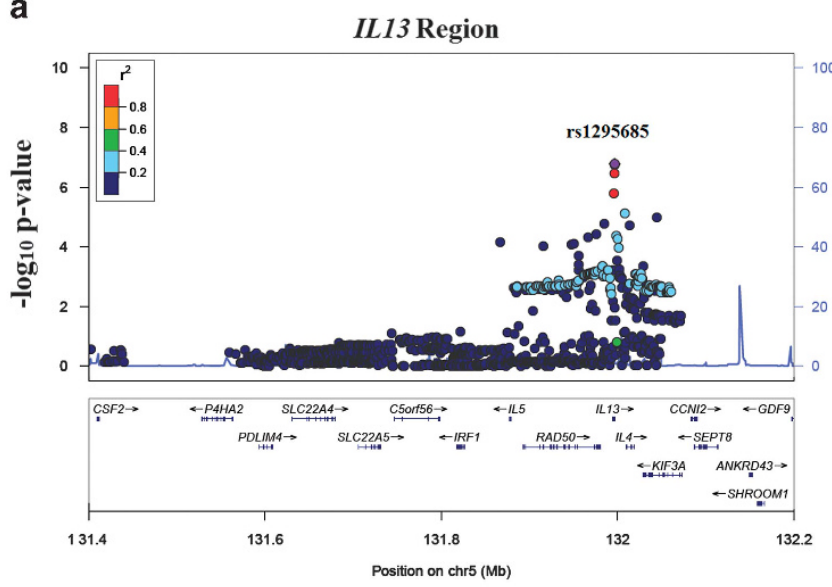

C

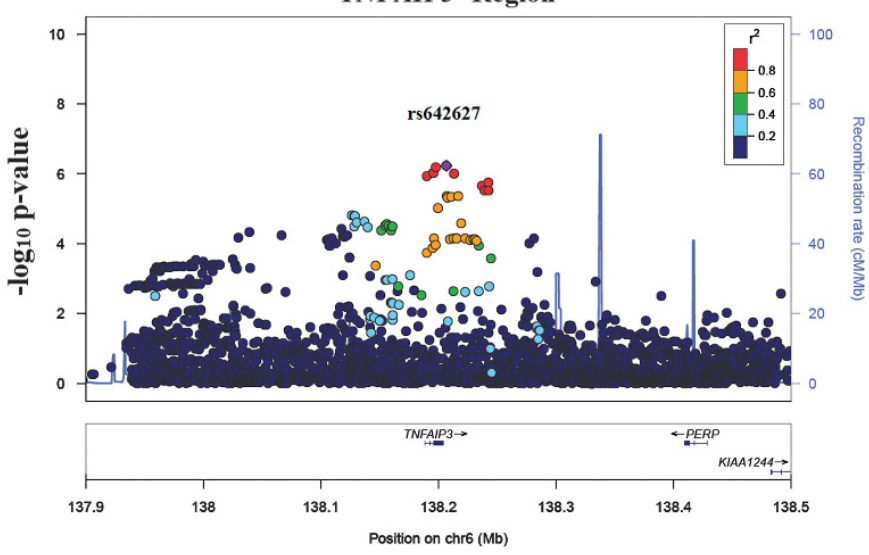

b

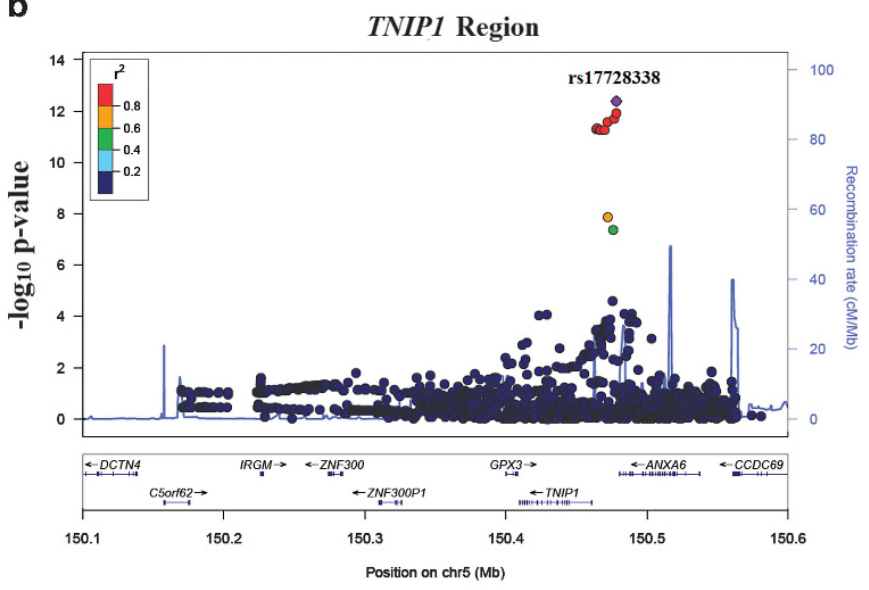

d

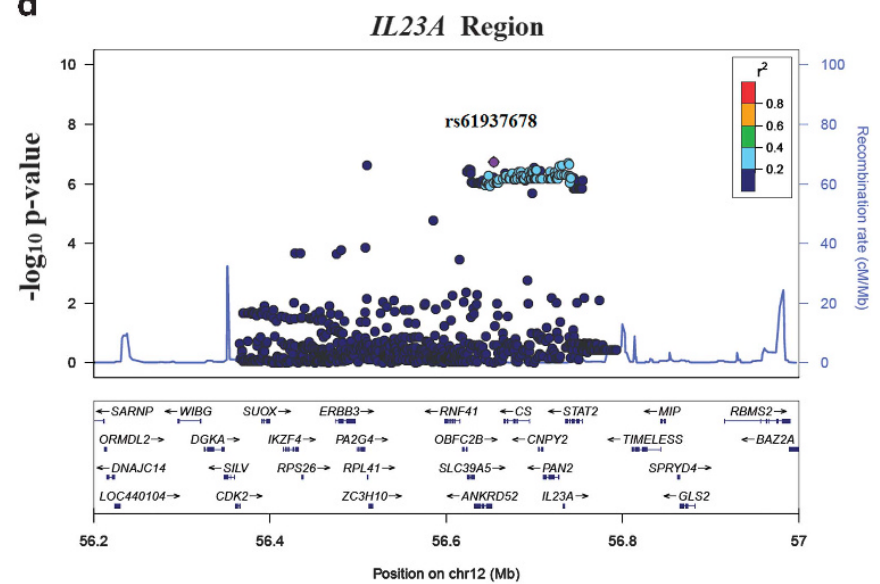

Figure 3 Regional association plot for IL13, TNIP1, TNFAIP3 and IL23A. Association plots for the IL13, TNIP1, TNFAIP3 and IL23A regions are shown in (a), (b), (c) and (d), respectively. The horizontal axis represents the base position of the corresponding region. The left-vertical axis represents the negative $\log$ (base 10) of the $P$-value in the corresponding round (denoted by the circles) and the right-vertical axes represents the recombination rate (in $\mathrm{cM} / \mathrm{Mb}$ ) (denoted by the blue lines). The color coding for the circles is used to represent the linkage disequilibrium with the most significant SNP in the region in each round.

Table 3 Comparison of results from this study with Tsoi et al

\begin{tabular}{|c|c|c|c|c|c|c|}
\hline Region & Base position & SNP identifier & Base position & SNP identifier & $\begin{array}{c}\text { Haplotype } \\
\text { based LD }\left(r^{2}\right)\end{array}$ & $\begin{array}{c}\text { Distance } \\
\text { (in kb units) }\end{array}$ \\
\hline IL13 & 131996445 & Rs1295685 & 131996445 & Rs1295685 & 1 & 0 \\
\hline IL12B & 158804928 & Rs4379175 & 158766022 & Rs62377586 & 0.84 & 38.91 \\
\hline TNIP1 & 150467189 & Rs2233278 & 150478318 & Rs17728338 & 0.96 & 11.13 \\
\hline TNFAIP3 & 138197824 & Rs582757 & 138206783 & Rs642627 & 0.97 & 8.9 \\
\hline \multirow[t]{2}{*}{$\mathrm{MHC}$} & 31266090 & Rs4406273 & 31316699 & Rs114255771 & 1 & 50.6 \\
\hline & 31355119 & Rs13437088 & 31358302 & Rs6924962 & 0.56 & 3.18 \\
\hline
\end{tabular}

Base positions are based on build 37/hg 19

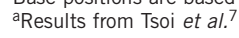





Figure 4 Comparison of results from this study with results from Knight et $a^{\beta}$ and Feng et al. ${ }^{12}$ Comparison of the results from this study with the results from Knight et $a^{\beta}$ and Feng et al ${ }^{12}$ is shown in (a) and (b), respectively. The SNPs on the vertical axes represent the significant SNPs from this study and the SNPs on the horizontal axes represent the significant SNPs from Knight et $a^{\beta}$ and Feng et $a^{12}$ in (a) and (b), respectively. The blocks (corresponding to each SNP on the horizontal axes versus each SNP on the vertical axes) with the color coding represents the strength of linkage disequilibrium between the corresponding SNPs, which was calculated as a measure to show corroboration between the studies at hand.

in normal human epidermal keratinocytes (NHEKs) (Supplementary Table 3). While a few of the putative intergenic variants lie within transcribed regions identified in the GM12878 cell line, none of them lie within conserved genomic regions (using phastCons 44-way alignments). Some variants are within enhancer regions based on $\mathrm{H} 3 \mathrm{~K} 4 \mathrm{Me} 1, \mathrm{H} 3 \mathrm{~K} 4 \mathrm{Me} 3$ and $\mathrm{H} 3 \mathrm{~K} 27 \mathrm{Ac}$ chromatin marks in the GM12878 cell line, and a total of eight variants in TNIP1, IL12B, MHC and TNFAIP3 are located in strong enhancers in NHEKs. Variants in TNIP1, IL13, IL12B, TNFAIP3 and the primary MHC signal lie within DNase I-hypersensitive sites, but no variants lie within CTCF (zinc-finger protein) binding sites. Moreover, eight variants in IL13, TNIP1 and TNFAIP3 lie within transcription factor binding sites delineated by ChIP-Seq data from the ENCODE project. ${ }^{30}$

To assess potential causal variants in the MHC region, we investigated the squared Pearson's correlations between imputed dosages of the three MHC SNP signals from this study and dosages of 2-digit resolution alleles of six MHC genes (HLA-A, -B, -C, -DRB1, $-D Q B 1, M I C A)$ that were genotyped on a subset of 1429 Canadian individuals from our sample. ${ }^{32}$ The risk allele of each of these signals is tagged best by an allele of a nearby gene (Table 2), with $r^{2}$ between rs114255771 and HLA- $C^{\star} 06=0.90$, between rs6924962 and $M I C A^{\star} 002=0.34$ and between rs892666 and $H L A-A^{\star} 03=0.38$. $H L A-A^{*} 03$ is positively correlated with the protective $\mathrm{T}$ allele of rs892666; the strongest squared correlation of the $\mathrm{C}$ risk allele of this SNP among all 2-digit HLA alleles is with $H L A-A^{\star} 02\left(r^{2}=0.16\right)$.

We also imputed 126 classical 2-digit alleles, 298 4-digit alleles and 1276 polymorphic amino-acid residues of eight HLA genes (HLA-A, $-B,-C,-D P A 1,-D P B 1,-D Q A 1,-D Q B 1$ and $-D R B 1)$, and combined these with more than 40000 imputed SNP and indel variants in the $\mathrm{MHC}$ region. The combined variant set was then subjected to stepwise forward logistic regression to identify independent association signals and to determine the relationship of these potentially causative HLA gene variants with the best SNP and indel signals in the MHC region. As shown in Supplementary Table 4, the two best independent signals from this analysis with significant $P$-values after Bonferroni correction (rs114255771, rs6924962) are the same two variants identified in the previous analysis of SNPs and indels only (Table 2); the third best signal (rs1059514 chr6.hg19. g.29911190G $>$ A $)$ is in moderate LD $\left(r^{2}=0.41\right)$ with the third best signal (rs892666) in the previous analysis.

We identified $H L A-C^{\star} 06: 02$ as the most strongly allied proteincoding variant for the first SNP signal (rs114255771), which is consistent with evidence presented just above (Supplementary Table 4). However, rs114255771 shows association of much greater strength and significance $\left(\mathrm{OR}=4.04 ; P\right.$-value $\left.=2.6 \times 10^{-66}\right)$ than either $H L A-C^{\star} 06: 02\left(\mathrm{OR}=2.84 ; P\right.$-value $\left.=1.9 \times 10^{-55}\right)$ or $\mathrm{rs} 4406273$ $\left(\mathrm{OR}=2.85 ; \quad P\right.$-value $\left.=2.0 \times 10^{-55}\right)$, a near-perfect surrogate for HLA-C $C^{\star} 06: 02$. This is an unexpected result, because SNPs rs114255771 and rs4406273 score as in complete LD $\left(r^{2}=1.000\right)$ in the 379 individuals of European descent in the phase I 1000 genomes data set. We hypothesized that this discrepancy may be caused by suboptimal imputation quality of rs114255771 in our analysis sample (predicted $r^{2}$ of imputed and actual genotyped dosages $=0.752$ ), whereas rs4406273 is genotyped for our sample, leading to an observed $r^{2}$ between rs114255771 and rs4406273 of 0.899 rather than 1.000. We tested this hypothesis using a test sample of 5393 cases and controls with empirically determined genotypes for rs114255771, rs4406273 and $H L A-C^{\star} 06: 02 ; 2761$ individuals in the test sample overlap the sample analyzed by this study. LD among these three variants in the test sample was very high $\left(r^{2}=0.980-0.989\right)$, and their associations with psoriasis were very similar $(\mathrm{OR}=3.19-3.24$; $P$-value $\left.=3.8 \times 10^{-77}-1.8 \times 10^{-75}\right)$. From these results, it is clear that if rs114255771 had been genotyped in the analysis sample its association with psoriasis would have been nearly identical to that seen for $H L A-C^{\star} 06: 02$ or rs4406273.

Although the superior association of rs114255771 in the stepwise analysis can be accounted for as an artifact of its suboptimal imputation, it appears to be driven by a genuine genetic effect. In our sample, the imputed risk allele of rs 114255771 is more strongly correlated with genotyped alleles of the extended ancestral haplotype $H L A-A^{\star} 02: 01-C^{*} 06: 02-B^{\star} 57: 01-M I C A^{*} 017-D R B 1^{*} 07: 01-D Q B 1^{\star} 03: 03$ than are the genotyped risk allele of rs4406273 or $H L A-C^{\star} 06: 02$ itself (data not shown). Thus, the stronger tagging by rs114255771 of the HLA- $C^{\star} 06: 02-B^{\star} 57: 01$ haplotype appears to be because of it being more accurately imputed for this haplotype than for other $H L A-C^{\star} 06: 02$-bearing haplotypes. Whatever the reason, the better 
Table 4 Summary of association for different MHC haplotype groups

\begin{tabular}{|c|c|c|c|c|c|c|c|c|}
\hline \multirow[b]{2}{*}{ Haplotype group } & \multicolumn{4}{|c|}{ Individual odds ratios } & \multicolumn{4}{|c|}{ Difference in log odds ratio with $C * 06-B * 57$ haplotypes } \\
\hline & Odds ratio & $P$-value & Frequency cases & Frequency controls & $\log$ odds & Difference & $S E$ & $\mathrm{P}$-value \\
\hline HLA-C*06 allele & 2.84 & $1.92 \mathrm{E}-55$ & 0.210 & 0.090 & 1.046 & 0.114 & 0.079 & 0.150 \\
\hline HLA-C*06 without $B * 57$ & 2.07 & $1.02 \mathrm{E}-18$ & 0.108 & 0.054 & 0.727 & 0.433 & 0.136 & 0.002 \\
\hline HLA-C*06-B*57 haplotype & 3.19 & $1.02 \mathrm{E}-33$ & 0.105 & 0.036 & - & - & - & - \\
\hline HLA-B*57 without $C * 06$ & 1.61 & 0.18 & 0.005 & 0.002 & 0.480 & 0.680 & 0.393 & 0.083 \\
\hline HLA-B*57 allele & 3.08 & $6.77 \mathrm{E}-34$ & 0.100 & 0.039 & 1.125 & 0.035 & 0.026 & 0.178 \\
\hline
\end{tabular}

The differences in odds ratios, when compared with the $H L A-C^{*} 06-B^{*} 57$ haplotype, were also estimated and tested for significance. Bootstrap was used to calculate standard error. Bold represents those haplotype groups that have a significant difference than $H L A-C^{*} 06-B^{*} 57$ haplotype.

tagging of $H L A-C^{\star} 06-B^{\star} 57$ results in a stronger disease association for imputed rs114255771 than for $H L A-C^{\star} 06$, because, as shown in Table 4, the $H L A-C^{\star} 06-B^{\star} 57$ haplotype is indeed more strongly associated with psoriasis than are other $H L A-C^{\star} 06$-bearing haplotypes $(\mathrm{OR}=3.19$ vs 2.07; $P$-value for difference in $\log \mathrm{ORs}=0.002)$. The estimated ORs of the various haplotype groups in Table 4 suggest a multiplicative model where the $H L A-C^{\star} 06-B^{\star} 57$ haplotype is hypothesized to increase risk for psoriasis by the product of the ORs of the individual $H L A-C^{\star} 06$ and $H L A-B^{\star} 57$ alleles (joint OR of $3.19 \approx$ OR of 2.07 for $H L A-C^{\star} 06$ without $H L A-B^{\star} 57 \times$ OR of 1.61 for $H L A-B^{\star} 57$ without $\left.H L A-C^{\star} 06\right)$. However, given the low frequency of $H L A-B^{\star} 57$ haplotypes lacking $H L A-C^{\star} 06$ (0.005 cases/0.002 controls), a larger sample would be needed to test this hypothesis.

\section{DISCUSSION}

Using a custom-designed SNP array, we conducted a fine-mapping study of eight loci known to be associated with psoriasis, confirming associations and revealing multiple independent association signals in the $\mathrm{MHC}$ and $I L 12 B$ regions. Because this experiment did not encompass all subsequently described data sets, its power to detect conditional signals was limited. Nevertheless, this study serves as a largely independent corroboration of the findings of a recent large meta-analysis of psoriasis, ${ }^{7}$ as only 145 samples are shared between the two studies (representing $2.9 \%$ of the samples in this study and $0.4 \%$ of those in the meta-analysis). Five of the association signals identified by the two studies (IL13, TNIP1, TNFAIP3, primary MHC and the primary $I L 12 B$ signal in this study and secondary $I L 12 B$ signal in the GWAS-Immunochip meta-analysis ${ }^{7}$ ) are in strong LD between studies $\left(r^{2}=0.84-1.00\right)$ and explain a very similar amount of the variance in disease liability $(7.45 \%$ in this study $v s 7.78 \%$ in the metaanalysis) (Table 3 ). Hence, these signals likely tag the same underlying causative variants. However, three other pairs of association signals (the unconditional IL23A signals, the first conditional signals in the MHC, and our conditional IL12B signal vs the primary IL12B in the recent meta meta-analysis ${ }^{7}$ ) are in weaker $\mathrm{LD}$ between studies $\left(r^{2}=0.20-0.56\right)$, showing very different amounts of the variance in liability (3.54\% in this study $v s \quad 1.31 \%$ in the meta-analysis), and could thus be tagging different disease variants.

Our results for the MHC region provide a completely independent replication of two previous fine-mapping studies of this same region. 8,12 Although the primary signals agree with each other across all three studies, the conditional signals of similar rank are not in high LD with each other. Our third signal from MHC (rs892666) does not exhibit high LD with any previously identified MHC signal, but lies only $7.8 \mathrm{~kb}$ from the fourth signal of Knight et $a l^{8}$ study. One explanation for the differences among studies is that we have high power for correctly tagging the very strong HLA-Cw06 signal as the most important risk locus in all the three studies, but we have lesser power for detecting and correctly ordering the more weakly associated secondary signals. Thus, we would expect unanimity in selecting the primary signals but variation in the identity and rank order of other signals until sample sizes become very large.

Adding imputed protein alleles and polymorphic amino-acid residues for eight HLA genes to the SNPs and indels in the association analysis of the MHC region revealed that SNP rs114255771 tags $H L A-C^{\star} 06: 02$, which has been shown by multiple studies as the leading candidate for the PSORS1 locus in the MHC. ${ }^{10,11}$ Although all three SNP signals in the MHC are allied with coding variants of nearby genes, our finding remains that the SNPs are more strongly associated with disease than the putative causative gene variants. For the best SNP signal, this stronger association was demonstrated to be an artifact of reduced imputation quality. The greater strength of association of the other two SNP signals compared with the best HLA variants could also be a result of imputation, especially considering that most of the SNPs were imputed using a different reference panel than was used for imputing the HLA variants. Alternatively, we cannot rule out the possibility that the causative variants underlying the association of these other two SNPs affect MHC genes other than the eight HLA genes included in our analysis, especially given that SNP2HLA does not impute the HLA-like MICA alleles.

Analysis of imputed HLA haplotypes demonstrated that the $H L A-C^{\star} 06-B^{\star} 57$ haplotype is more strongly associated with psoriasis than are other $H L A-C^{\star} 06$-bearing haplotypes. This differential association has been suggested previously, albeit in much smaller studies, ${ }^{10,12,33}$ but to our knowledge this is the first time the difference in strength of association has been shown to be statistically significant.

All eight loci were densely genotyped and further enhanced by imputation, enabling us to study rare and common variants including indels that might provide clues about disease mechanisms or increase the heritability explained by these loci. ${ }^{34}$ Still, none of the best signals identified by the conditional analyses were either indels or rare variants. In total, the nine association signals obtained from this study passing the multiple-testing threshold explain $12.09 \%$ of the overall variance in liability for psoriasis or $\sim 18.3 \%$ of its estimated heritability.

We searched for causal markers in our 39 significant variants and their proxies (Supplementary Table 3) and found only one nonsynonymous SNPS (rs20541), which is a proxy (LD $r^{2}=0.97$ ) for our best IL13 association signal (rs1295685). The minor A allele of rs20541 changes amino-acid 130 of $I L-13$ from arginine (R) to glutamine $(\mathrm{Q})$, and has been associated with increased IgE levels and risk for asthma, ${ }^{35}$ and reduced risk of helminth infections. ${ }^{36}$ Notably, this variant has been shown to be more active than the 130R wildtype allele in inducing STAT6 phosphorylation and activation. ${ }^{37,38}$ Consistent with the important role of Th17 cells in psoriasis 
pathogenesis $^{39}$ and the antagonistic effects of IL13 on IL17 production by human Th17 lymphocytes, ${ }^{40}$ this allele exerts a protective effect against psoriasis. ${ }^{7}$ Most of the signals detected in this study might be regulatory as they fall outside the genes and conserved regions, but many fall in transcribed regions, DNase I-hypersensitive sites and transcription factor binding sites. Similar results have recently been reported for rheumatoid arthritis ${ }^{41}$ and several other complex genetic disorders. ${ }^{42-45}$ We used chromHMM predictions to find that some of our variants lie within enhancer regions and are strong enhancers for NHEK cell lines. As the principal cell of the epidermis, NHEK represents, by far, the majority cell type present in the skin and which is greatly expanded in psoriasis lesion, relative to normal skin. ${ }^{7,46}$ Moreover, there is evidence from functional assays that the HLA-Cw6 association may relate to altered expression of this gene owing to altered enhancer function, rather than variation in its antigen-presenting properties. ${ }^{47,48}$ Additionally, a number of recent papers attest to tissue-specific phenotypes that arise from selective silencing of TNFAIP3 in various mouse cell types. ${ }^{49}$

Our study was too small for signals near IL23R and RNF114 to reach genome-wide significance. However, we did find nominally significant association for the already established signals in these two regions, rs9988642 chr1.hg19.g.67726104T >C (IL23R) and rs495337 chr20.hg19.g.48522330C > T (RNF114).,6,9 SNP rs495337 was genotyped in our data and had a $P$-value of $9.20 \times 10^{-5}$, whereas the SNP rs9988642 was imputed (estimated imputation $r^{2}=0.96$ ) and had a $P$-value of $1.46 \times 10^{-5}$. Notably, rs9988642 is in strong LD with the rs11209026 chr1.hg19.g.67705958G > A variant, ${ }^{7}$ which specifies glutamine at residue 381 in the IL-23 receptor, is associated with reduced risk of psoriasis, and abrogates signaling through the receptor. ${ }^{50,51}$

In conclusion, we provide evidence for the existence of multiple signals in previously known loci associated with psoriasis, replicating and extending the findings of other fine-mapping studies in a largely independent sample, thereby refining the candidate regions for each association signal. The MHC region explains more variance than all other loci combined, and even the secondary MHC signal explains more variance than any other variant (or locus). Our analysis further indicates that $H L A-C, M I C A$ and $H L A-A$ are good candidates for the three MHC signals identified on the basis of SNP associations. Outside the MHC, our studies provide clues to the potential causative mechanisms of the association signals (eg, the two IL12B signals are likely non-exonic and therefore regulatory and not protein-changing, whereas the IL13 signal may exert its effect through a nonsynonymous variation with effects on IL13 protein function), and annotation analyses have identified plausible mechanisms for regulatory mechanisms of noncoding variations. Whatever be the mechanisms involved, the observation that each of the loci achieving significance in this study has a plausible role in immunosurveillance ${ }^{7}$ strongly supports further functional exploration of psoriasis-associated genetic variation in the context of host defense.

\section{CONFLICT OF INTEREST}

The authors declare no conflict of interest.

\section{ACKNOWLEDGEMENTS}

We thank all the psoriasis patients and normal controls who contributed samples to this study. This work was supported by NIH Grants R01AR042742, R01AR054966, R01AR050511, R01AR050266 and R01AR062382. The University of Toronto Psoriatic Arthritis Program is supported by the Krembil Foundation and the CIHR. JTE is supported by the Ann Arbor Veterans Affairs Hospital. This research uses resources provided by the Type 1 Diabetes
Genetics Consortium (T1DGC); a collaborative clinical study sponsored by the National Institute of Diabetes and Digestive and Kidney Diseases (NIDDK); National Institute of Allergy and Infectious Diseases (NIAID); National Human Genome Research Institute (NHGRI); National Institute of Child Health and Human Development; Juvenile Diabetes Research Foundation International (JDRF), supported by U01 DK062418.

1 Elder JT, Bruce AT, Gudjonsson JE et al: Molecular dissection of psoriasis: integrating genetics and biology. J Invest Dermatol 2010; 130: 1213-1226.

2 Yates VM, Watkinson G, Kelman A: Further evidence for an association between psoriasis, Crohn's disease and ulcerative colitis. Br J Dermatol 1982; 106: 323-330.

3 Ellinghaus D, Ellinghaus E, Nair RP et al: Combined analysis of genome-wide association studies for Crohn disease and psoriasis identifies seven shared susceptibility loci. Am J Hum Genet 2012; 90: 636-647.

4 Nair RP, Duffin KC, Helms $\mathrm{C}$ et al: Genome-wide scan reveals association of psoriasis with IL-23 and NF-kappaB pathways. Nat Genet 2009; 41: 199-204.

5 Genetic Analysis of Psoriasis Consortium, Wellcome Trust Case Control Consortium 2 Strange A et al: A genome-wide association study identifies new psoriasis susceptibility loci and an interaction between HLA-C and ERAP1. Nat Genet 2010; 42: 985-990.

6 Stuart PE, Nair RP, Ellinghaus E et al: Genome-wide association analysis identifies three psoriasis susceptibility loci. Nat Genet 2010; 42: 1000-1004.

7 Tsoi LC, Spain SL, Knight J et al: Identification of 15 new psoriasis susceptibility loci highlights the role of innate immunity. Nat Genet 2012; 44 1341-1348.

8 Knight J, Spain SL, Capon F et al: Conditional analysis identifies three novel major histocompatibility complex loci associated with psoriasis. Hum Mol Genet 2012; 21 . 5185-5192.

9 Capon F, Bijlmakers MJ, Wolf $\mathrm{N}$ et al: Identification of ZNF313/RNF114 as a novel psoriasis susceptibility gene. Hum Mol Genet 2008; 17: 1938-1945.

10 Nair RP, Stuart PE, Nistor I et al: Sequence and haplotype analysis supports HLA-C as the psoriasis susceptibility 1 gene. Am J Hum Genet 2006; 78: 827-851.

11 Fan X, Yang S, Huang $W$ et al: Fine mapping of the psoriasis susceptibility locus PSORS1 supports HLA-C as the susceptibility gene in the Han Chinese population. PLoS Genet 2008; 4: e1000038.

12 Feng BJ, Sun LD, Soltani-Arabshahi R et al: Multiple Loci within the major histocompatibility complex confer risk of psoriasis. PLoS Genet 2009; 5: e1000606.

13 Genomes Project CAbecasis, Auton A et al: An integrated map of genetic variation from 1,092 human genomes. Nature 2012; 491: 56-65.

14 Carlson CS, Eberle MA, Rieder MJ, Yi Q, Kruglyak L, Nickerson DA: Selecting a maximally informative set of single-nucleotide polymorphisms for association analyses using linkage disequilibrium. Am J Hum Genet 2004; 74: 106-120.

15 Thomson W, Barton A, Ke X et al: Rheumatoid arthritis association at 6q23. Nat Genet 2007; 39: 1431-1433.

16 Plenge RM, Cotsapas C, Davies L et al: Two independent alleles at 6q23 associated with risk of rheumatoid arthritis. Nat Genet 2007; 39: 1477-1482.

17 Abecasis GR: RelativeFinder. Software Package.

18 Li Y, Willer CJ, Ding J, Scheet P, Abecasis GR: MaCH: using sequence and genotype data to estimate haplotypes and unobserved genotypes. Genet Epidemiol 2010; 34 816-834.

19 Howie B, Fuchsberger C, Stephens M, Marchini J, Abecasis GR: Fast and accurate genotype imputation in genome-wide association studies through pre-phasing. Nat Genet 2012; 44: 955-959.

20 Jia X. Han B, Onengut-Gumuscu S et al: Imputing amino acid polymorphisms in human leukocyte antigens. PLoS One 2013; 8: e64683.

21 Kang HM, Sul JH, Service SK et al: Variance component model to account for sample structure in genome-wide association studies. Nat Genet 2010; 42: 348-354.

22 Devlin B, Roeder K: Genomic control for association studies. Biometrics 1999; 55 997-1004.

23 Purcell S, Neale B, Todd-Brown $\mathrm{K}$ et al: PLINK: a tool set for whole-genome association and population-based linkage analyses. Am J Hum Genet 2007; 81: 559-575.

24 Falconer DS: The inheritance of liability to certain diseases, estimated from the incidence among relatives. Ann Hum Genet 1965; 29: 51-76.

25 So HC, Gui AH, Cherny SS, Sham PC: Evaluating the heritability explained by known susceptibility variants: a survey of ten complex diseases. Genet Epidemiol 2011; 35 310-317.

26 So HC, Sham PC: Effect size measures in genetic association studies and age-conditional risk prediction. Hum Hered 2010; 70: 205-218.

27 Wang K, Li M, Hakonarson H: ANNOVAR: functional annotation of genetic variants from high-throughput sequencing data. Nucleic Acids Res 2010; 38: e164.

28 Karolchik D, Barber GP, Casper J et al: The UCSC Genome Browser database: 2014 update. Nucleic Acids Res 2014; 42: D764-D770.

29 Pruitt KD, Brown GR, Hiatt SM et al: RefSeq: an update on mammalian reference sequences. Nucleic Acids Res 2014; 42: D756-D763.

30 Consortium EP. The ENCODE (ENCyclopedia Of DNA Elements) Project. Science 2004; 306: 636-640.

31 Ernst J, Kellis M: ChromHMM: automating chromatin-state discovery and characterization. Nat Methods 2012; 9: 215-216. 
32 Pollock R, Chandran V, Barrett J et al: Differential major histocompatibility complex class I chain-related A allele associations with skin and joint manifestations of psoriatic disease. Tissue Antigens 2011; 77: 554-561.

33 Jenisch S, Henseler T, Nair RP et al: Linkage analysis of human leukocyte antigen (HLA) markers in familial psoriasis: strong disequilibrium effects provide evidence for a major determinant in the HLA-B/-C region. Am J Hum Genet 1998; 63: 191-199.

34 Manolio TA, Collins FS, Cox NJ et al: Finding the missing heritability of complex diseases. Nature 2009; 461: 747-753.

35 Potaczek DP, Kabesch M: Current concepts of IgE regulation and impact of genetic determinants. Clin Exp Allergy 2012; 42: 852-871.

36 Grant AV, Araujo MI, Ponte EV et al: Functional polymorphisms in IL13 are protective against high Schistosoma mansoni infection intensity in a Brazilian population. PLoS One 2012; 7: e35863.

37 Andrews AL, Bucchieri F, Arima $\mathrm{K}$ et al: Effect of $\mathrm{IL}-13$ receptor alpha2 levels on the biological activity of IL-13 variant R110Q. J Allergy Clin Immunol 2007; 120: 91-97.

38 Vladich FD, Brazille SM, Stern D, Peck ML, Ghittoni R, Vercelli D: IL-13 R130Q, a common variant associated with allergy and asthma, enhances effector mechanisms essential for human allergic inflammation. J Clin Invest 2005; 115: 747-754.

39 Lowes MA, Russell CB, Martin DA, Towne JE, Krueger JG: The IL-23/T17 pathogenic axis in psoriasis is amplified by keratinocyte responses. Trends Immunol 2013; 34: 174-181.

40 Newcomb DC, Boswell MG, Huckabee MM et al: IL-13 regulates Th17 secretion of IL-17A in an IL-10-dependent manner. J Immunol 2012; 188: 1027-1035.

41 Okada Y, Wu D, Trynka G et al: Genetics of rheumatoid arthritis contributes to biology and drug discovery. Nature 2013; 506: 376-381.
42 International Multiple Sclerosis Genetics CBeecham, Patsopoulos NA et al: Analysis of immune-related loci identifies 48 new susceptibility variants for multiple sclerosis. Nat Genet 2013; 45: 1353-1360.

43 Parkes M, Cortes A, van Heel DA, Brown MA: Genetic insights into common pathways and complex relationships among immune-mediated diseases. Nat Rev Genet 2013; 14: 661-673.

44 Liu JZ, Almarri MA, Gaffney DJ et al: Dense fine-mapping study identifies new susceptibility loci for primary biliary cirrhosis. Nat Genet 2012; 44: 1137-1141.

45 Manku H, Langefeld CD, Guerra SG et al: Trans-ancestral studies fine map the SLE-susceptibility locus TNFSF4. PLoS Genet 2013; 9: e1003554.

$46 \mathrm{Li} \mathrm{B}$, Swindell R, Gudjonsson J et al: Transcriptome analysis of psoriasis in a large case-control sample: RNA-seq provides insights into disease mechanisms. J Invest Dermatol 2013; 134: 1828-1838.

47 Clop A, Bertoni A, Spain SL et al: An in-depth characterization of the major psoriasis susceptibility locus identifies candidate susceptibility alleles within an HLA-C enhancer element. PLoS One 2013; 8: e71690.

48 Hundhausen C, Bertoni A, Mak RK et al: Allele-specific cytokine responses at the HLA-C locus: implications for psoriasis. J Invest Dermatol 2012; 132: 635-641.

49 Martin F, Dixit VM: A20 edits ubiquitin and autoimmune paradigms. Nat Genet 2011; 43: 822-823.

$50 \mathrm{Di}$ Meglio P, Di Cesare A, Laggner $U$ et al: The IL23R R381Q gene variant protects against immune-mediated diseases by impairing IL-23-induced Th17 effector response in humans. PLoS One 2011; 6: e17160.

51 Di Meglio P, Villanova F, Napolitano L et al: The IL23R A/GIn381 allele promotes IL-23 unresponsiveness in human memory T-helper 17 cells and impairs Th17 responses in psoriasis patients. J Invest Dermatol 2013; 133: 2381-2389.

Supplementary Information accompanies this paper on European Journal of Human Genetics website (http://www.nature.com/ejhg) 